\title{
ON A CHARACTERIZATION OF THE LATTICE OF SUBSYSTEMS OF A TRANSITION SYSTEM
}

\author{
J. P. MAVOUNGOU AND C. NKUIMI-JUGNIA
}

Received 4 October 2005; Revised 20 May 2006; Accepted 28 May 2006

It was first proved by Birkhoff and Frink, and the result now belongs to the folklore, that any algebraic lattice is up to isomorphism the lattice of subuniverses of a universal algebra. A study of subsystems of a transition system yields a new algebraic concept, that of a strongly algebraic lattice. We give here a representation theorem to the manner of Birkhoff and Frink of such lattices.

Copyright (c) 2006 Hindawi Publishing Corporation. All rights reserved.

A transition system is a pair $\left(S, \longrightarrow_{S}\right)$, where

(i) $S$ is a set of states,

(ii) $\longrightarrow_{S} \subseteq S \times S$ is the transition relation.

We write $s \longrightarrow_{S} s^{\prime}$ for $\left(s, s^{\prime}\right) \in \longrightarrow_{S}$.

Nondeterministic transition systems, those $\left(S, \longrightarrow_{S}\right)$ for which the set of successors of any element $s \in S$ is an arbitrary set, are easily seen to be coalgebras of the covariant powerset functor $\mathscr{P}:$ Sets $\rightarrow$ Sets from the category of sets to itself.

Observe that any unary algebra $(S, \mathscr{F})$ gives rise to a unique transition system $\left(S, \longrightarrow_{S}\right)$, but the converse in the general case is false.

A subsystem of a transition system $\left(S, \longrightarrow_{S}\right)$ is a subset $X$ of $S$ which has the following stability property: $s \longrightarrow_{S} s^{\prime}$ and $s \in X$ imply $s^{\prime} \in X$. The empty set and the universe $S$ are subsystems of $\left(S, \longrightarrow_{S}\right)$, they are said to be trivial. It is straightforward to check that the set $\operatorname{Subs}(S)$ of subsystems of $\left(S, \longrightarrow_{S}\right)$ is stable for arbitrary unions and intersections. Given a subset $X$ of $S$, we denote by $\langle X\rangle$ the subsystem of $\left(S, \longrightarrow_{S}\right)$ generated by $X$. It is the intersection of all subsystems of $\left(S, \rightarrow_{S}\right)$ containing $X$. The notation $\underset{\rightarrow_{S}}{*}$ will be used to denote the reflexive and transitive closures of the binary relation $\longrightarrow_{S}$ on $S$. The 
subsystem $\langle X\rangle$ is then characterized as follows:

$$
\langle X\rangle=\left\{x^{\prime} \in X: \exists x \in X, x \stackrel{*}{\longrightarrow_{S}} x^{\prime}\right\}
$$

Hence for $s \in S$, writing $\langle s\rangle$ the subsystem $\langle\{s\}\rangle$, we get

$$
\langle s\rangle=\left\{s^{\prime} \in S: s \stackrel{*}{\longrightarrow} s^{\prime}\right\}
$$

The mapping $\langle-\rangle: \mathscr{P}(S) \rightarrow \mathscr{P}(S)$ defined from the set of subsets of $S$ to itself is a closure operator on $S$. The previous characterization of $\langle X\rangle$ permits to see that

$$
\langle X\rangle=\left\{x^{\prime} \in S: \exists x \in X, x^{\prime} \in\langle x\rangle\right\}=\bigcup_{x \in X}\langle x\rangle .
$$

We say that the closure operator $\langle-\rangle$ is completely additive. One can notice that

(i) subsystems $\langle s\rangle$ of $\left(S, \vec{S}_{S}\right), s \in S$, satisfy the following finiteness condition: for all families $\left(X_{i}, i \in I\right)$ of subsystems of $\left(S, \longrightarrow_{S}\right)$ if $\langle s\rangle \subseteq \bigcup_{i \in I} X_{i}$, then there exists an index $i_{0} \in I$ such that $\langle s\rangle \subseteq X_{i_{0}}$,

(ii) $\left\langle s^{\prime}\right\rangle \subseteq\langle s\rangle$ if and only if $s \stackrel{*}{\longrightarrow} s^{\prime}$.

These observations prompt us to initiate the following definitions.

Definition 1. Let $(E, \leq)$ be an ordered set which admits arbitrary suprema. An element $a$ in $E$ is called $s$-compact ( $s$ for strongly compact), if for all covering $a \leq \bigvee_{i \in I} a_{i}$ of $a$ there exists an index $i$ for which $a \leq a_{i}$.

Consider a sup-complete lattice $(E, \leq)$ (i.e., an ordered set admitting arbitrary supre$\mathrm{ma})$. As a poset, $(E, \leq)$ can be viewed as a cocomplete category. Let $a$ be in $E$, it is equivalent to say that $a$ is $s$-compact or in categorical terms, every morphism $f: a \rightarrow \operatorname{colim}_{I} a_{i}$ factors uniquely into a morphism $\bar{f}: a \rightarrow a_{i}$ (for some $i \in I$ ). This means that the covariant hom-functor $[a,-]$ preserves all (small) colimits. Such an object $a$ is called absolutely presentable (see [2]).

Definition 2. A sup-complete lattice $(L, \leq)$ is called $s$-algebraic (or strongly algebraic), if each element $a$ of $L$ can be written as supremum of $s$-compact elements less than $a$.

Any s-algebraic lattice is obviously algebraic, but the converse is not true. In fact given a group $(G, *)$, the lattice $(\operatorname{Sg}(G), \subseteq)$ of subgroups of $G$ is algebraic (see [1]). Further algebraic elements in $(\operatorname{Sg}(G))$ are finitely generated subgroups of $G$. It is easy to verify that $(\operatorname{Sg}(\mathbb{Z},+), \subseteq)$ the lattice of subgroups of the additive group $(\mathbb{Z},+)$ is not $s$-algebraic.

Consider the sup-complete lattice $(L, \leq)$ as a cocomplete category; it will be called $s$ algebraic if every element in $L$ is a colimit of absolutely presentable objects in $L$. Hence an $s$-algebraic lattice viewed as a category is locally absolutely presentable with the set of $s$-compact elements as set of absolutely presentable objects.

The basic example is that of a complete lattice of subsystems of a transition system; this seems also to be a generic $s$-algebraic lattice as shown by the following representation theorem. 
Theorem 3. Let $(L, \leq)$ be an s-algebraic lattice. There exist a transition system $\left(S, \rightarrow_{S}\right)$ and an isomorphism from $L$ onto the lattice $\operatorname{Subs}(S)$ of subsystems of $\left(S, \rightarrow_{S}\right)$.

Proof. We denote by $S$ the set of $s$-compact elements of $L$. Define on $S$ a binary relation $\vec{S}_{S}$ as follows: for all $a, b \in S, a \longrightarrow_{S} b$ if and only if $b \leq a$. Let $\downarrow x$ be the set of elements $x^{\prime} \in L$ such that $x^{\prime} \leq x$. For all $x$ in $L$, the set $S \cap \downarrow x$ of $s$-compact elements less than $x$ is a subsystem of $S$. In fact if $a \longrightarrow_{S} b$ and $a \in S \cap \downarrow x$, then we have $b \leq a$, hence $b \in S \cap \downarrow x$. On deduces the mapping

$$
\psi: L \longrightarrow \operatorname{Subs}(S), \quad x \longmapsto S \cap \downarrow x
$$

Let us check that $\psi$ is order preserving and reflecting. To this end, let us consider $x$ and $x^{\prime}$ in $L$. If $x \leq x^{\prime}$, then $\downarrow x \subseteq \downarrow x^{\prime}$ and therefore $S \cap \downarrow x \subseteq S \cap \downarrow x^{\prime}$, that is, $\psi(x) \subseteq \psi\left(x^{\prime}\right)$. Conversely if $\psi(x) \subseteq \psi\left(x^{\prime}\right)$, since each element of $L$ can be written as a supremum of $s$-compact elements less than itself, we have $x=\bigvee \psi(x) \leq \bigvee \psi\left(x^{\prime}\right)=x^{\prime}$.

Finally let us show that $\psi$ is a one-to-one mapping by exhibiting its inverse. For that set the mapping

$$
\phi: \operatorname{Subs}(S) \longrightarrow L, \quad X \longmapsto \bigvee X
$$

For all $x \in L$, we have $\phi \psi(x)=\bigvee\{a \mid a$ is $s$-compact and $a \leq x\}=x$. Further, for all subsystem $X$ of $\left(S, \longrightarrow_{S}\right)$,

$$
\psi \phi(X)=\psi(\vee X)=S \bigcap \downarrow \bigvee X
$$

It is clear that $X \subseteq S \cap \downarrow \vee X$. Let $a \in L$ such that $a \leq \vee X$ and $a \in S$. By $s$-compacity of $a$, there exists $x \in X$ such that $a \leq x$, that is, $x \longrightarrow_{S} a$ by definition. Since $X$ is a subsystem of $\left(S, \longrightarrow_{S}\right)$ and $x \in X$, we obtain $a \in X$. One deduces the inclusion $S \cap \downarrow \vee X \subseteq X$ which induces the equality $S \cap \downarrow \vee X=X$, hence $\psi \phi(X)=X$.

The fact that $\psi$ preserves arbitrary suprema follows from the fact that each order isomorphism between complete lattices is automatically an isomorphism of complete lattice. The theorem is proved.

Since the $s$-algebraic lattice $(L, \leq)$ as a poset is a locally absolutely presentable category, it is isomorphic to the free cocompletion $\left[S^{0}, S e t\right]$ of the set $S$ of $s$-compact elements, under all (small) colimits. This free cocompletion is, of course, isomorphic to the lattice of down-closed subsets of $S$ which are precisely the subsystems of $S$. Therefore Theorem 3 gives a theoretical lattice version of the categorical well-known result stating that: every locally absolutely presentable category is isomorphic to the presheaf category.

\section{Acknowledgments}

We wish to thank referees for useful advice and remarks. 


\section{The lattice of subsystems of a transition system}

\section{References}

[1] S. Burris and H. P. Sankappanavar, A Course in Universal Algebra, Graduate Texts in Mathematics, vol. 78, Springer, New York, 1981.

[2] C. Centazzo, J. Rosický, and E. M. Vitale, A characterization of locally D-presentable categories, Cahiers de Topologie et Géométrie Différentielle Catégoriques 45 (2004), no. 2, 141-146.

J. P. Mavoungou: Department of Mathematics, Faculty of Science, University of Yaoundé 1, P.O. Box 812, Yaoundé, Cameroun

E-mail address: jpmavoungou@yahoo.fr

C. Nkuimi-Jugnia: Department of Mathematics, Faculty of Science, University of Yaoundé 1, P.O. Box 812, Yaoundé, Cameroun

E-mail address: nkuimi@yahoo.co.uk 


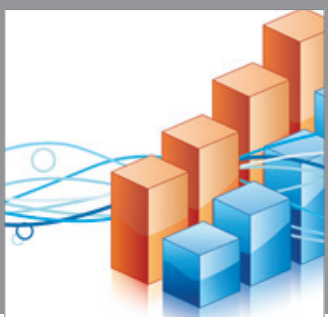

Advances in

Operations Research

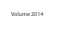

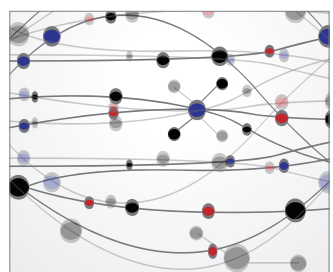

\section{The Scientific} World Journal
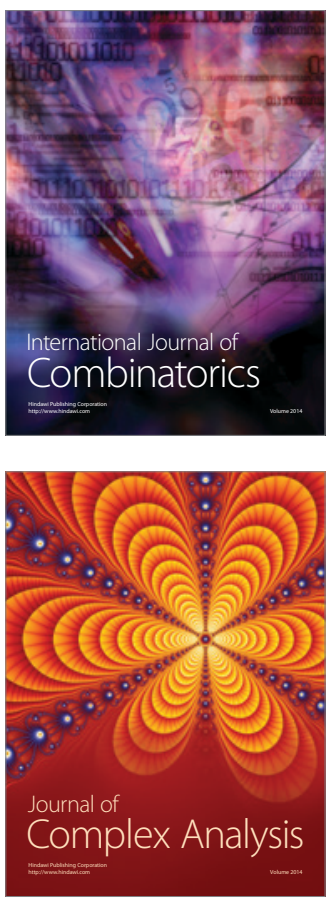

International Journal of

Mathematics and

Mathematical

Sciences
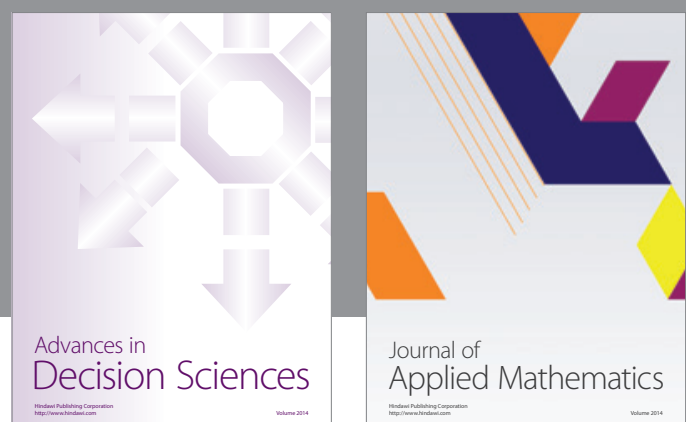

Journal of

Applied Mathematics
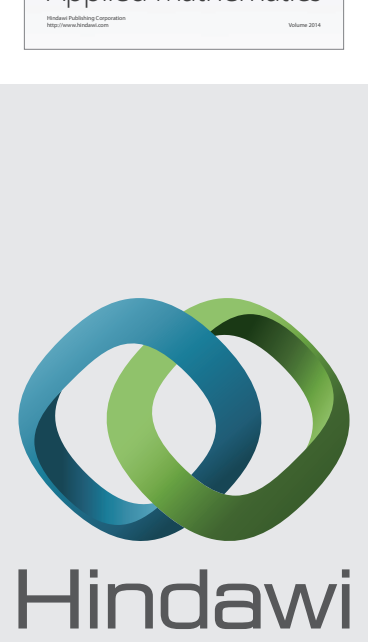

Submit your manuscripts at http://www.hindawi.com
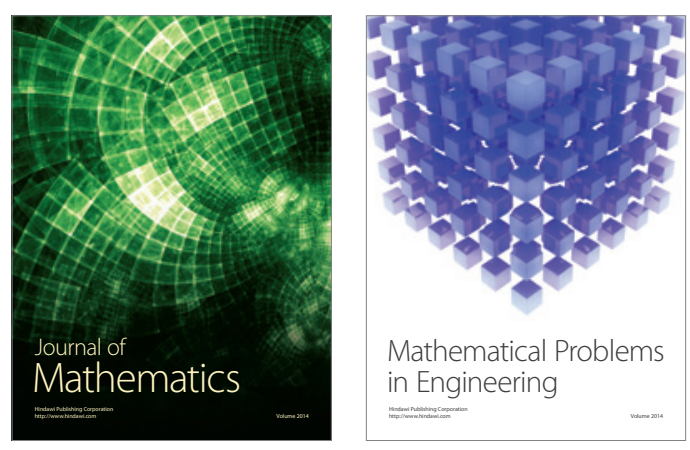

Mathematical Problems in Engineering
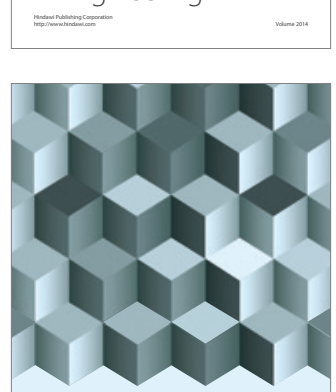

Journal of

Function Spaces
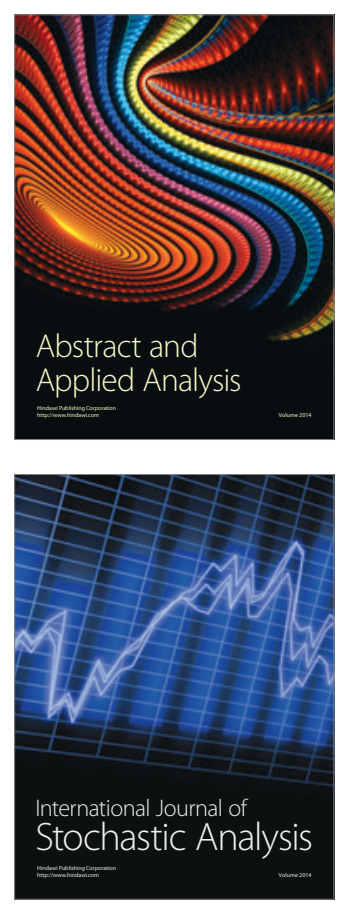

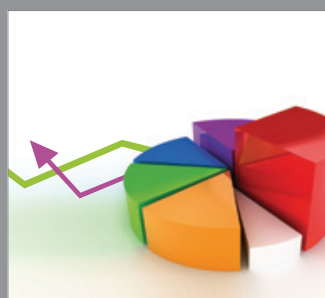

ournal of

Probability and Statistics

Promensencen
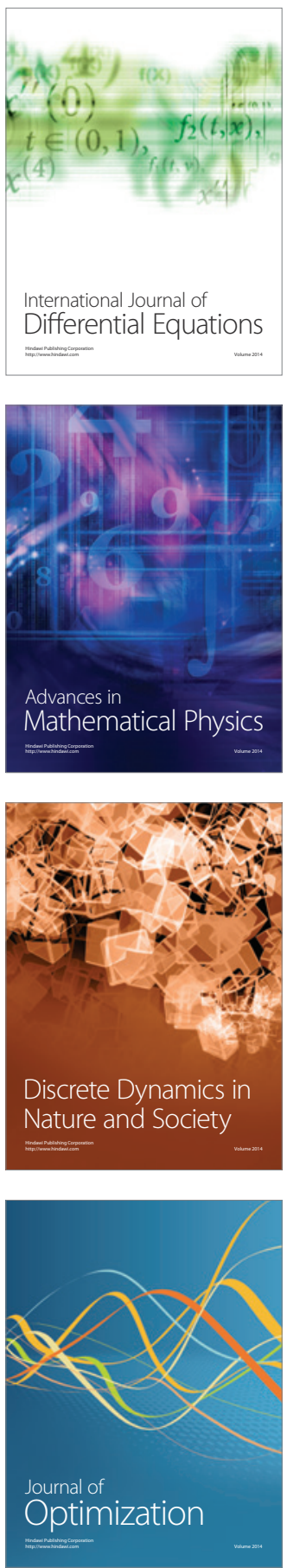